\title{
Efficient routing on small complex networks without buffers
}

\author{
J. Smiljanić, I. Stanković \\ ${ }^{a}$ Scientific Computing Laboratory, Institute of Physics Belgrade, University of Belgrade, Pregrevica 118, 11080 Belgrade, Serbia
}

\begin{abstract}
In this paper, we are exploring strategies for the reduction of the congestion in the complex networks. The nodes without buffers are considered, so, if the congestion occurs, the information packets will be dropped. The focus is on the efficient routing. The routing strategies are compared using two generic models, i.e., Barabàsi-Albert scalefree network and scale-free network on lattice, and the academic router networks of the Netherlands and France. We propose a dynamic deflection routing algorithm which automatically extends path of the packet before it arrives at congested node. The simulation results indicate that the dynamic routing strategy can further reduce number of dropped packets in a combination with the efficient path routing proposed by Yan et al. [Phys. Rev. E 73, 046108 (2006)].
\end{abstract}

Keywords: scale-free networks, routing, network capacity

\section{Introduction}

Complex networks are important for functioning of the modern society. To ensure a free, uncongested traffic flows on the complex networks is of great interest. Intuitively, the traffic congestion could be largely reduced or completely avoided with a very large average degree of connectivity and/or node capacity for information packet delivery. The capacity of nodes to deliver information cannot be infinite. Also, upgrading the infrastructure is often not economically feasible [1, 2]. The performance of the communication systems can be improved by implementing the more appropriate routing protocols without changing the underlying network structure [3-17], which is more realizable in the practice. Such work presents two problems. The first is finding out the optimal strategies for the traffic routing on a defined network structure. The second problem is finding a procedure to draw general conclusions about performance of routing strategies due to the variation of the real network topologies. The increasing speed of the network interfaces raises an important question concerning the size of buffers, complexity and cost. A considerable research effort is currently under way in an attempt to resolve compromise between buffer latency and complexity on one side and capacity on the other [18-25]. In the previous studies, the node buffer size in the traffic-flow model is set as infinite [3-16]. Our intention is to produce a relatively simple methodology for evaluating routing strategies in networks with limited buffering capability or without optical buffers. This should be important for the optical networks with either large volumes of the information traffic or without buffering capacity.

A number of network models is introduced in the last two decade [26, 27]. A particular class of models is dedicated to networks embedded in the space [28]. Here we are interested in evaluating the models for representation of the spatially constrained networks, both in terms distance between nodes and the extent of the network. We are interested in information flow optimization in the small networks. The small networks should represent bulk of telecommunication networks or other dedicated information networks, e.g., regional optical backbones, academic networks. As examples of the the real-world networks, we analyze the national research and educational networks (NRENs) of the Netherlands [29], France [30], Norway [31] and Spain [32] and compare them with Barabàsi-Albert scale-free network and scale-free network on lattice. The system size dependence of topological characteristics of the scale-free network on lattice is also analyzed.

Email address: jelena.smiljanic@ipb.ac.rs (J. Smiljanić) 
In this work three routing strategies are implemented and evaluated: the shortest path routing, efficient path routing and dynamic deflection routing. The shortest path routing is widely used routing strategy in praxis (by "shortest" we mean the path with the smallest number of links) [33]. However, in the shortest path routing strategy load distribution is not homogeneous. The majority of the shortest paths pass through the nodes that are highly connected, while other nodes carry much less traffic [34]. Yan et al. [5] presented an approach to redistribute traffic load in highly connected nodes to other nodes using link weight. An improvement is achieved through a targeted traffic redistribution from the most congested nodes. As result the congestion is reduced at expense of a slight increase of the total path length and traffic. We compare this routing strategy with a dynamic routing strategy. The dynamic routing strategy improves the control of the congestion in the heavily loaded nodes by dynamically returning packet one step back. In this way, the dynamic strategy uses the redundant capacity of the links in network to temporarily store information, until congested node capacity is free. Further, we test a possibility of combining dynamic and static routing strategy. In order to check how different routing strategies behave in the larger networks, we have evaluated information loss dependence on packet generation rate and network size in case of the scale-free network on lattices.

The paper is organized as follows: in Sec.II we introduce the generic scale-free model and the scale-free model on lattice and compare their network characteristics with the national research and educational networks (NRENs) of the Netherlands, France, Norway and Spain. Furthermore, the characteristics of the scale-free model on lattice for different system sizes are considered. In Sec.III, the information flow model and a measure of system performance are introduced. The static and dynamic routing strategies are described in Sec.IV and their performance is analyzed in Sec.V.

\section{Network models}

In this work, we compare topological network characteristics of Barabàsi-Albert scale-free model [26], the scalefree model on lattice [28] and the national research and educational networks (NRENs) of the Netherlands, France, Norway and Spain. Barabàsi and Albert observed an existence of a high degree of self-organization characterizing the large-scale properties of complex networks [26]. They have introduced a model of the scale-free networks with two key elements: probability that a new node connects to the existing nodes is not uniform and there is a higher probability that it will be linked to a node that already has a large number of connections. Thus, Barabàsi-Albert (BA) scale-free network model is formed in a series of steps in which new nodes are incorporate into the network. Algorithm is starting with a small number $\left(N_{0}\right)$ of nodes, and at every time step new nodes with $m$ connections are added. To incorporate preferential attachment, the model assumes that the probability of the new connection with the node $i$ depends on its connectivity $k_{i}$ and equals $P\left(k_{i}\right)=k_{i} / \sum k_{j}$. After a few algorithm steps, distribution of number of links per node takes scale-free form $P(k) \sim k^{-\lambda}$. In this work, for $N_{0}=3$ and $m=2, n=61$ algorithm steps are preformed. Obtained network consists of $N=64$ nodes with $\lambda=2$. The obtained network degree distribution corresponds well to the NRENs, cf. Fig. 1(a) The number of nodes in different NRENs is in the case of the Netherlands $N=59$, France $N=63$, Norway $N=58$ and Spain $N=53$.

However, the real-life networks are embedded into the geographical space and constrained by the cost of the links between the nodes. In the scale-free model on lattice, cf. [28], the algorithm starts with a set of nodes that are identified with the set of lattice vertices in an $M \times M$ square. The lattice distance between two nodes is defined as minimal number of "lattice steps" separating them in the regular lattice. In this model, network nodes are randomly assigned with the number of links $(k)$ according to scale-free distribution $P(k)=A k^{-\lambda}, m \leq k<K$ and connected to its closest neighbors. Therefore, exponent $\lambda$ is a model parameter. We set $\lambda=2$, as obtained from the connectivity distribution of NRENs, cf. Fig. 1(a) The choice of model parameter $\lambda$ is also in accordance with the distribution of the number of links per node obtained with BA model. Normalization constant is $A \approx(\lambda-1) m^{\lambda-1}$.

At the first glance the most efficient mean to transfer information through the network is along the shortest paths. The distribution of the shortest path lengths is given in Fig. 1(b). The network diameter $D$, can be defined as the maximal length of the shortest path between any two nodes in the network, i.e., $D=\max \left\{d_{i j}\right\}$, where length of shortest path from the node $i$ to the node $j$ is $d_{i j}$. The small network diameter means that packets transmitted through the network, travel from one node to another quickly along the shortest path. As result the possibility of loss due to the congestion of the transmitting nodes is reduced. From Fig. 1(b) one can observe that the path length distribution of the BA scale-free network does not match NRENs well. This is not surprising, since in the BA scale-free model the Euclidean distance between nodes is irrelevant. For the version of scale-free network on lattice, the model has 

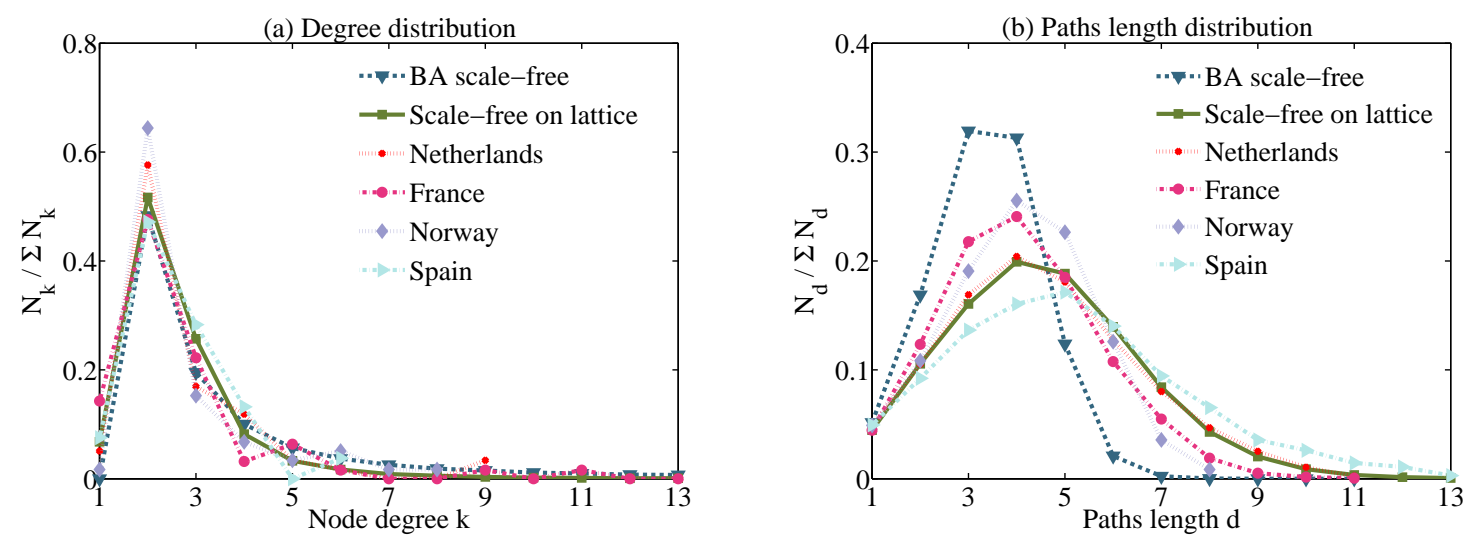

Figure 1: Topological characteristics: (a) degree distribution; (b) length of path probability density function for NRENs of the Netherlands $(N=59)$, France $(N=63)$, Norway $(N=58)$ and Spain $(N=53)$ and generic network models: BA scale-free network $(N=64, m=2)$ and scale-free network on lattice $(N=64, \lambda=2)$.

desirable properties in terms of path lengths. Network diameter in different NRENs is in the case of the Netherlands $D=11$, France $D=11$, Norway $D=8$ and Spain $D=13$. Scale-free network (BA) generation algorithm generates networks with considerably smaller network diameters $D=6.84$. Average network diameter obtained for scale-free on lattice model is $D=10.81$, and compares well with diameters of NRENs.

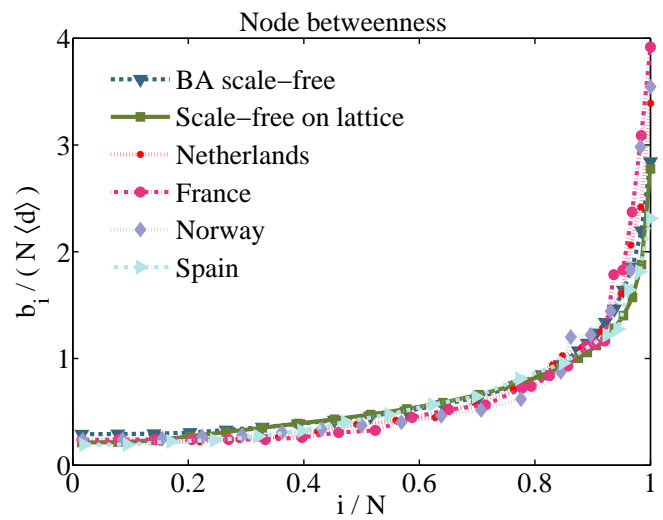

Figure 2: Distribution of normalized node betweenness for NRENs of the Netherlands $(N=59,\langle d\rangle=4.54)$, France $(N=63,\langle d\rangle=4.08)$, Norway $(N=58,\langle d\rangle=4.06)$ and $\operatorname{Spain}(N=53,\langle d\rangle=5.08)$ and generic network models: BA scale-free network $(N=64, m=2,\langle d\rangle=3.36)$ and scale-free network on lattice $(N=64, \lambda=2,\langle d\rangle=4.59)$.

The quality of the communication of the two non-adjacent nodes, i.e., node $j$ and node $k$, depends on the nodes belonging to the paths connecting the nodes $j$ and $k$. Consequently, a measure of the relevance of a given node for overall network performance can be obtained by counting the number of geodesics going through it, and defining the so-called node betweenness. More precisely, the betweenness $b_{i}$ of the node $i$, sometimes referred to also as load, is defined as [34, 35]:

$$
b_{i}=\sum_{j, k \in \mathcal{N}, j \neq k} n_{j k}(i)
$$

where $n_{j k}(i)$ is the number of the shortest paths connecting $j$ and $k$ and passing through the node $i$. Let $\langle d\rangle$ denote the average path length of the given network measured according to the shortest path routing rule. The normalized 
betweenness distribution for NRENs of the Netherlands, France, Norway and Spain and BA scale-free network and scale-free network on latticeis is shown in Fig. 2. We observe that both models reproduce betweenness characteristics of real networks well.
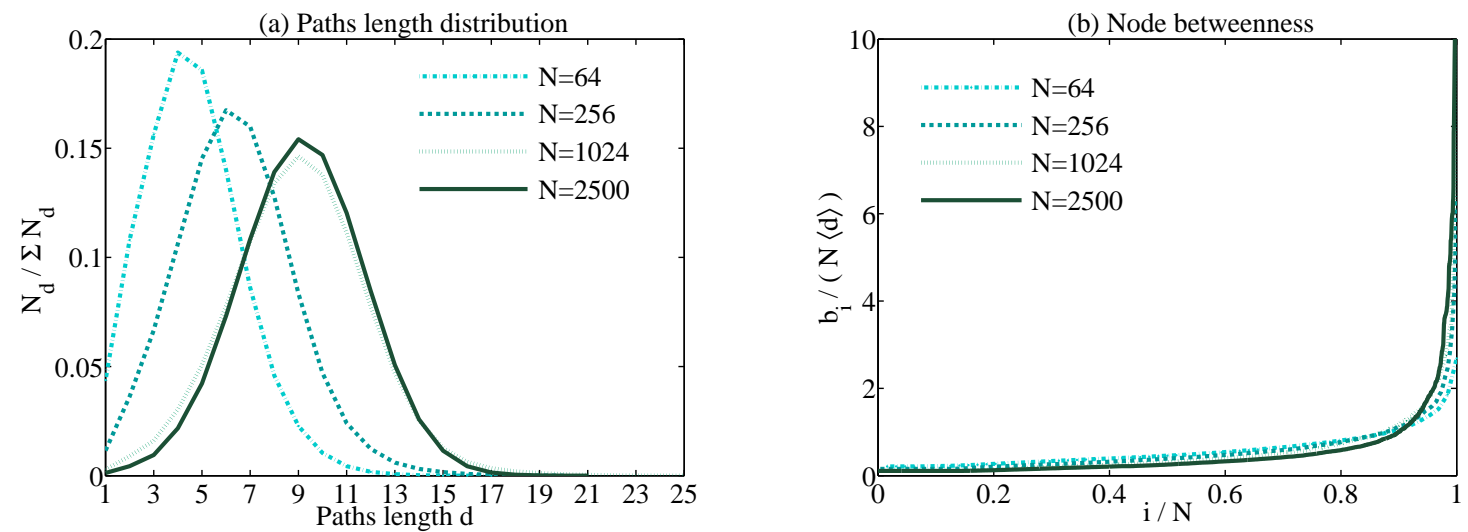

Figure 3: (a) Length of path probability density function for scale-free network on lattice for various system sizes $N$. (b) Distribution of normalized node betweeness for scale-free network on lattice for various system sizes $\mathrm{N}$, where $\langle d\rangle$ is average path length $(N=64,256,1024,2500$ and $\langle d\rangle=4.59,6.38,8.98,9.14$ respectively $)$.

In Fig. 3 we examine the system-size dependent $(N=64,256,1024$ and 2500$)$ behavior of the path length and node betweenness distribution for the scale-free on lattice model. The average path length initially increases fast with the system size (i.e., for $N<1024$ ). In the larger systems the path length distribution changes little with the system size, cf. $N=1024$ and 2500 in Fig. 3(a) The reason for this is existence of one or several nodes with high degree i.e. close to $N$, cf. Ref. [36]. The average betweenness is roughly proportional to the system size $N$ and the average path length $\langle d\rangle$ as $\left\langle b_{i}\right\rangle \sim N\langle d\rangle$. Also, the maximal value of node betweenness increases roughly with the square of system size $N$, cf. Ref. [34].

\section{Information flow model}

In the information flow model all nodes are treated as both hosts and routers. Each node has a predefined maximum packet routing capacity $C$ and communication channels have an infinite capacity to transmit the packets. If packets arrive to the node whose routing capacity has been already reached (i.e., congested node), they will be dropped. The dynamics of the model is as follows. At each time step $t$, an information packet is created at random node with the probability $p$. Therefore $p$ is the control parameter: small values of $p$ correspond to the uncongested (free) flow of packets and high values of $p$ correspond to the high flow rate of packets. When a new packet is created, a destination node, different from the origin, is chosen randomly in the network. In this paper, we analyze the case that each node is able to send one packet at each time step. The travel time $T$ of a packet is defined as the time spent by the packet between its source and destination. Here we do not take into account the time delay of the information transfer at each node or link, so that all data are delivered in a unit time, regardless of the distance between any two nodes. Thus, during the following time steps $t+1, t+2, \ldots, t+T$, the packet travels toward its destination and the time $T$ is related to the path length. Once the packet reaches the destination node, it is delivered and disappears from the network.

When the amount of packets is small, the network is able to deliver all the packets that are generated. Conversely, when $p$ is large enough the number of generated packets is larger than the number of packets that the network can manage to solve and the nodes enter in a state of congestion. The characteristic that measures the system performance is the packet drop probability $\eta$,

$$
\eta=\frac{R_{d}}{R}
$$

defined as a ratio of the total number of deleted packets $R_{d}$ and the total number of generated packets $R$. A high drop 
probability indicates that a large percentage of packets cannot reach their destinations. Then, the quality of service is poorer.

\section{Routing strategies}

\subsection{Static routing}

Packets can be delivered according to different routing strategies. When static weighted routing strategy is used, packets choose the routing path with the minimum sum weight of links. For any pair of source and destination node, there may be several paths with the same weight between them. We randomly choose one of these paths and put it into the fixed routing table which is followed by all packets. It has be assumed that each node has the same capability of delivering packets, that is, at each time step all the nodes can deliver at most $C$ packets one step toward their destinations according to the fixed routing table. Here we compare two static routing strategies: $(i)$ the shortest path routing, i.e., the links in the network have the same weight, $w_{s t}=1$, where $w_{s t}$ is weight of link going from $s$ to $t$. Routing communication along the shortest paths is of course beneficial for speed, but if there is a limit to the node load and network traffic is heavy, congestion is a threat to the nodes with the largest betweenness. Obviously, bypassing high-degree nodes, packet will have more chance to reach its destination. (ii) In the second, efficient path routing strategy, the weight of the link between the nodes $s$ and $t$ is defined as [5]:

$$
w_{s t}=\left(\frac{k_{s}+k_{t}}{\min _{i \neq j}\left(k_{i}+k_{j}\right)}\right)^{\beta} \text {, }
$$

where $k_{i}$ denotes degree of node $i$ and $\beta$ is an adjustable parameter. The efficient path between nodes $i$ and $j$ is corresponding to the route that makes the sum weight of links minimum. As for any pair of source and destination, there may be several efficient paths between them. We randomly choose one of them and put it into the fixed routing table which is followed by all the information packets.
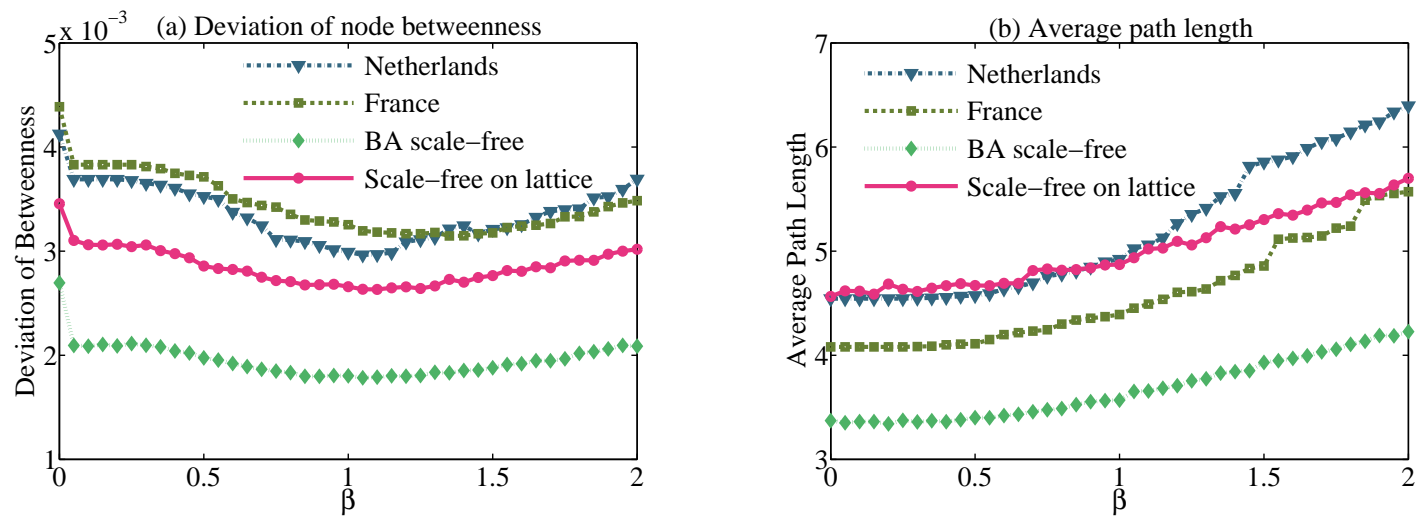

Figure 4: (a) Deviation of node betweenness vs parameter $\beta$ from Eq. 3 and (b) average path length vs parameter $\beta$ for different network models.

In Fig. 4(a), we show the betweenness deviation as a function of $\beta$ on different scale-free networks. The optimal routing strategy for generic scale-free network and scale-free network on lattice is corresponding to $\beta_{\text {opt }}=1 \pm 0.1$, where betweenness deviation is the smallest. This is also optima value for NREN of the Netherlands. The optimal value for French NREN is higher, i.e., $\beta_{\text {opt }}=1.2 \pm 0.1$, due to larger betweenness deviation. Obviously $\beta=0$ recovers the shortest path length. In comparison with the shortest path routing strategy average path length slightly increases with $\beta$, cf. Fig. 4(b). 


\subsection{Dynamic routing}

In this paper we also analyze the effectiveness of the dynamic deflection routing strategy. We assume each node has a knowledge about the load of its neighbors. If a package is about to arrive to a congested node, it will be deflected, i.e., its path will be dynamically extended. In other words, if we denote a path between nodes $i$ and $j$ as $P(i \rightarrow j):=x_{0}, x_{1}, \ldots, x_{n-1}, x_{n}$, where $x_{0}=i$ and $x_{n}=j$. The node $x_{m}$, instead forwarding packet to the congested node $x_{m+1}$, deflects the packet back to the node $x_{m-1}$. In the moment $t+1$, the node $x_{m-1}$ sends the packet to the node $x_{m}$, and in the moment $t+2$ the packet is sent to the node $x_{m+1}$. The deflection of the packet is tried only once.

The total number of the packets in network is equal to $p N\langle d\rangle$. Since node betweenness represents the number of paths going through the node, the probability that a packet in one time step is going through node $i$ is $B_{i}=\frac{b_{i}}{N(N-1)\langle d\rangle}$. Therefore, average number of packets going through node $i$ per step is $q_{i}=p N\langle d\rangle \times B_{i}=p \frac{b_{i}}{N-1}$. In order to avoid a reduction of the system performance due to the deflection traffic, we introduce a condition that a node can send packet back only if $q_{i}<0.5 C$. The localized congestion events are more likely to occur at the nodes with higher betweenness and that in the regime when traffic is not heavily congested. We find that it is sufficient to implement the deflection routing only in the nodes with highest betweenness (about $10 \%$ of all nodes). A higher amount of the nodes with deflection routing capability does not improve significantly the network performance.

\section{Simulation results}

To compare different routing strategies, we apply previously described routing algorithms and measure packet drop probability $\eta$ in the two generic networks and NRENs of France and the Netherlands. As shown in Fig. 5, the scale-free network on lattice under the shortest path routing strategy reproduces well behavior of both NREN topologies, for both simulated node transfer capacities $C=2$ and 4 . We can observe that in the real-world networks and scale-free network on lattice for $p>0.1$ and for node capacity $C=2$ more than $40 \%$ of generated packets is dropped. Also, when amount of the traffic in the network is small $(p<0.1)$, there is a significant amount of loss, i.e., more than $5 \%$. For the higher node transfer capacity $(C=4)$ number of deleted packets significantly decreases and there are almost no packets loss when the network traffic is small. The BA scale-free network is less prone to the congestion compared to the scale-free network on lattice and two NRENs, which is expected since this network has a shorter average path length.

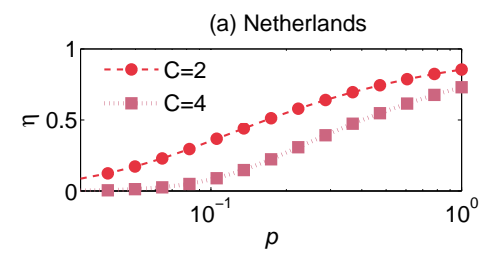

(c) BA scale-free

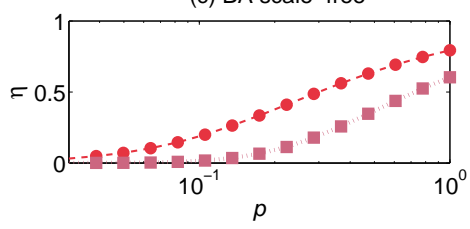

(b) France

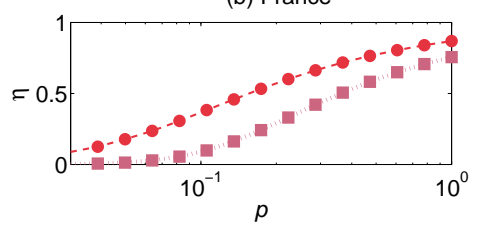

(d) Scale-free on lattice

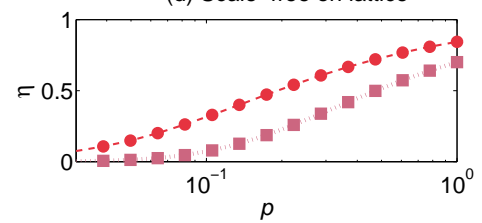

Figure 5: Packet drop probability $\eta$ vs. the packet-generating rate $p$ for the shortest path routing algorithm. We have set node transfer capacity $C=2$ (circles) and $C=4$ (squares).

Impact of the static and dynamic routing strategies is analyzed in Fig. 6 and Fig. 7 respectively. The results confirm that the static weighted routing using the topological information can greatly improve the traffic flow for small $p$ values $(p<0.1)$, in comparison with the shortest path routing mechanism. The static weighted routing method proves to be better for NREN topologies and scale-free model on lattice. The dynamic routing strategy is better in case of BA scale-free networks due to shorter paths, cf. Fig. 7. The shorter paths between nodes result in a 

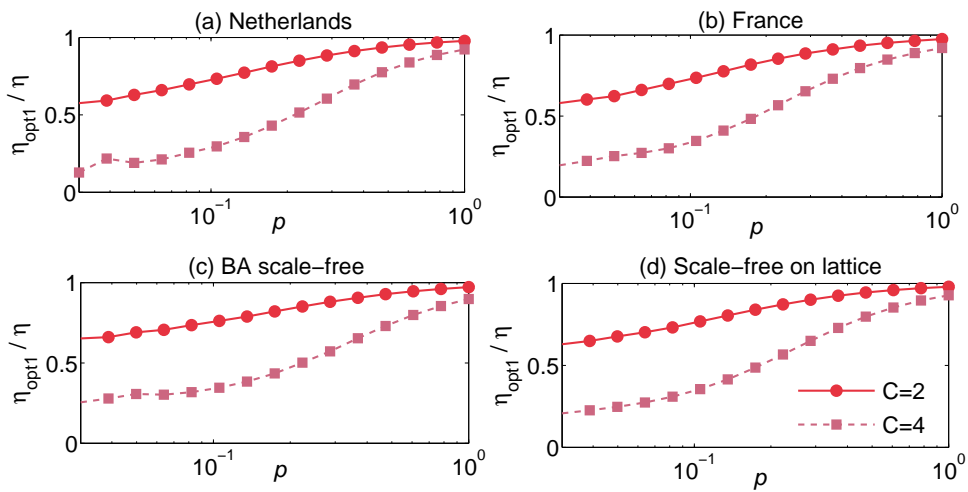

Figure 6: Comparison between packet drop probability $\eta$ as a function of packet-generating rate $p$ for the shortest path routing and static weighted routing strategy with $\beta_{\text {opt }}\left(\eta_{\text {opt } 1}\right)$. We have set node transfer capacity $C=2$ (circles) and $C=4$ (squares).
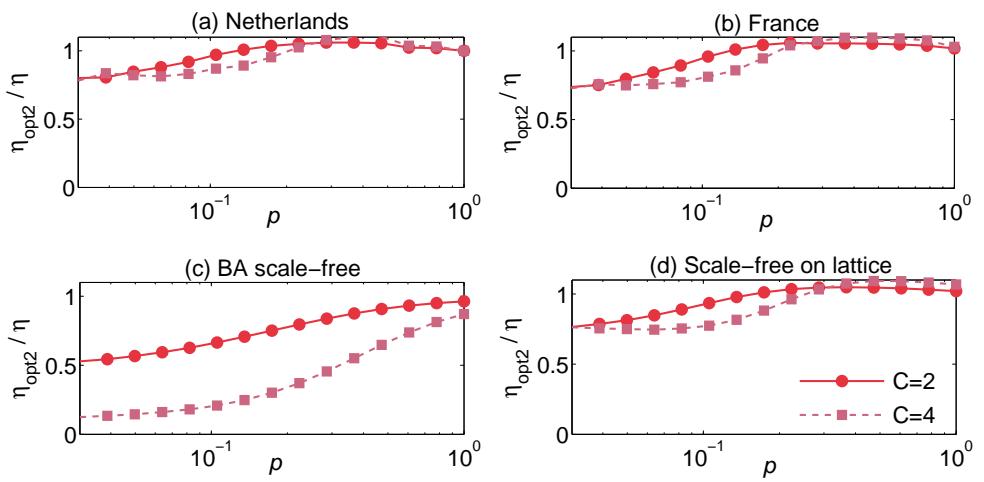

Figure 7: Comparison between packet drop probability $\eta$ as a function of packet-generating rate $p$ for the shortest path routing and dynamic routing algorithm $\left(\eta_{\text {opt } 2}\right)$. We have set node transfer capacity $C=2$ (circles) and $C=4$ (squares).

lower amount of the packages being transferred through the network at any instance of time compared to other studied networks, allowing for the dynamic deflection algorithm to be more efficient.

The dynamic and static routing strategies are complementary and can be combined. The system performance for combined static and dynamic routing and the static routing is compared in Fig. 8, The redistribution of the traffic load from the most congested nodes enables more efficient the dynamic deflection routing. Improvement is in range from $5 \%-20 \%$. In NREN of Netherlands four most congested nodes after path redistribution where in the proximity, and combined effect of dynamic-static routing strategy is rather weak, cf. Fig 8 a). On the other hand, the French NREN shows a considerable improvement due to the larger distance between the most congested nodes, cf. Fig 8 (b). This is consequence of the fact that both studied networks are small and the difference in the performance of the routing algorithms is a result of the variations in the topology. The scale-free model on lattice allows us to evaluate and compare routing strategy on large number of artificially generated networks. In this way we obtain information, how whole class of the networks responds to the routing strategy in average and we observe a considerable improvement in performance, cf. Fig. 8 (d).

Until now, we have tested the routing strategies for small networks (with $N \leq 64$ nodes). At this point, we want to show how the static and dynamic strategy behave in the larger networks, i.e., $N=256,1024$, and 2500. In Fig. 9 , we 

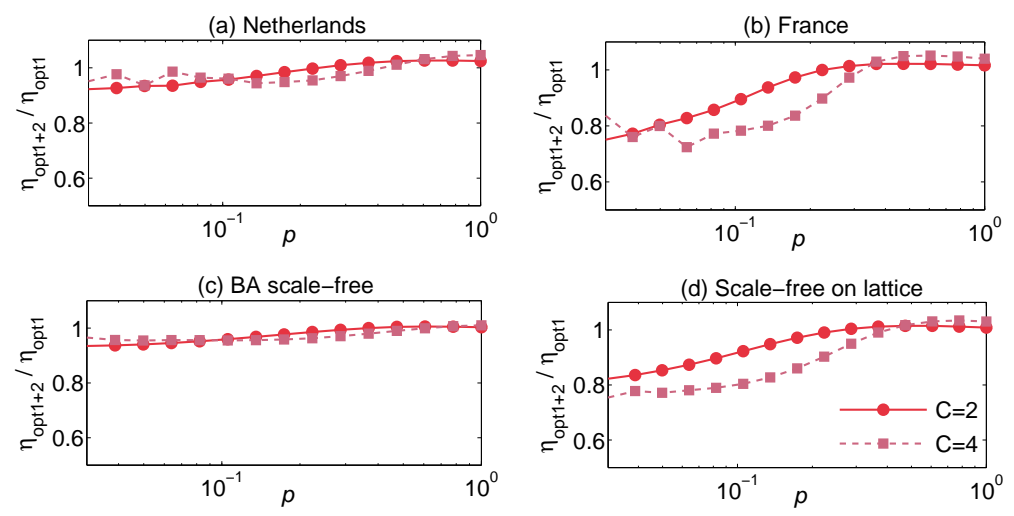

Figure 8: Comparison between packet drop probability $\eta$ as a function of packet-generating rate $p$ for static weighted routing strategy, $\beta_{\text {opt }}\left(\eta_{\text {opt } 1}\right)$ and combined static and dynamic routing $\left(\eta_{o p t 1+2}\right)$. We have set node transfer capacity $C=2$ (circles) and $C=4$ (squares).

show the relationship between packet drop probability $\eta$ and network size $N$ under different routing strategies on scalefree networks on lattice. In the case of the shortest path routing, the results are consistent with our intuition: with the increase of the system size $N$ the congestion starts at smaller packet generation rates $p$. In order to understand where the packet drop first occurs and now congestion develops, we need to look into the properties of the single network nodes. The probability of information drop (loss) on certain node depends on number of the packets going through it. The average number of packets going through node $i$ per step is $q_{i}=p \frac{b_{i}}{N-1}$ and the largest values of node betweenness, $b_{\max }$, increases faster then linear with system size, cf. Ref. [34] and Fig. 3(b)] Therefore increase of system size $N$ results in increase of drop probability. Fig. 9 b) compares the relation of the packet drop probability $\eta$ vs packetgenerating rate $p$ under the static weighted routing and the shortest path routing with different network size $N$. The efficiency of static weighted routing strategy increases with the system size $N$. Also, the larger networks compared to very small ones, i.e., $N=64$, offer more possibility to redistribute load and therefore the strategy is more efficient. On the other hand, dynamic routing algorithm changes alone is less efficient in larger networks, see Fig. 9(c). The reason for this is existence of the nodes with the large node degree (comparable to size of the network), cf. Ref. [36]. As result the number of deflected packets increases with the network size increasing congestion in the surrounding nodes. This problem is alleviated in the combination of the static weighted and dynamic routing algorithms, cf. Fig. 9 d). Since in this case the most congested nodes are avoided and the load is more evenly distributed, the dynamic routing can therefore further reduce congestion. We observe that improvement of network performance due to combined dynamic-static routing strategy is increasing with system size.

\section{Conclusions}

In summary, we have introduced a information flow model for networks without buffering capacity. We have shown that scale-free model on lattice reproduces well both topological and information transport characteristics of the small national research and educational networks of the Netherlands, France, Norway and Spain. In the small networks, the point of the network congestion and amount of information lost strongly depends on the underlying network structure. We have further described a dynamic deflection routing strategy suitable for the networks without buffers. The proposed strategy dynamically extends packet path before it reaches a congested node. Simulations on two real world networks and two generic networks with small diameter confirmed the highest traffic capacity under combined static and dynamic routing is achieved when compared with the shortest path routing strategy and the static weighted routing strategy. Moreover, we found that the dynamic deflection routing can further improve network information transport capacity when combined with the efficient path routing strategy both in small and large networks. 

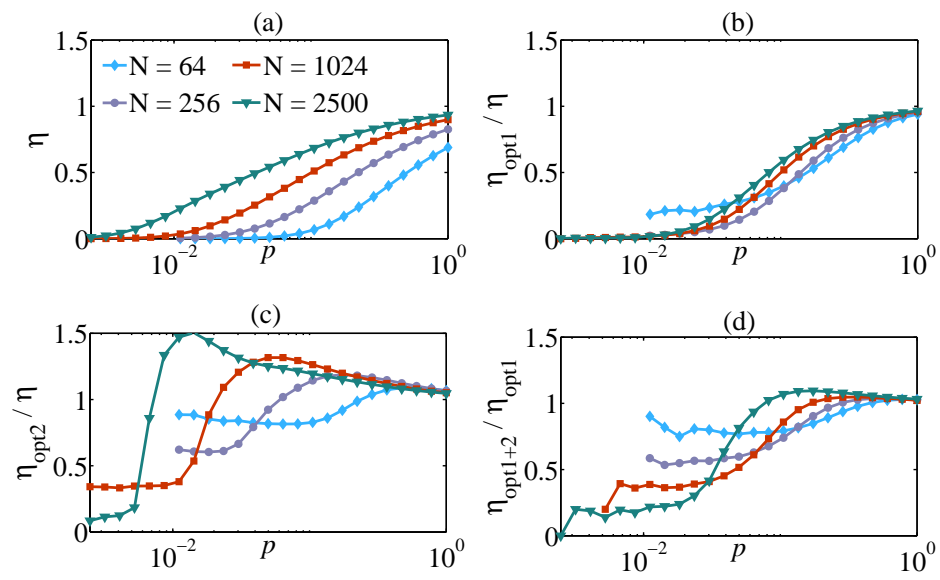

Figure 9: Packet drop probability $(\eta)$ in four scale-free on lattice networks with different sizes $N=64,256,1024$ and 2500. Results for different routing strategies are compared: (a) the shortest path routing algorithm, (b) static weighted routing strategy and shortest path routing $\left(\eta_{\text {opt } 1} / \eta\right)$, (c) dynamic routing algorithm and shortest path routing $\left(\eta_{\text {opt } 2} / \eta\right)$, and (d) combined static and dynamic routing strategy and static weighted routing strategy $\left(\eta_{\text {opt } 1+2} / \eta_{\text {opt } 1}\right)$. The value of node transfer capacity used in this figure is fixed $(C=4)$.

\section{Acknowledgements}

Discussions with Jovan Radunović and Vladica Tintor helped to motivate this effort. The authors acknowledge support by the Ministry of Science of the Republic of Serbia, under project No. ON171017. Numerical simulations were run on the AEGIS e-Infrastructure, supported in part by FP7 projects EGIInSPIRE, PRACE-1IP, PRACE-2IP, and HP-SEE. The authors also acknowledge support received through SCOPES grant IZ73Z0-128169 of the Swiss National Science Foundation.

\section{References}

[1] D.-H. Kim and A. E. Motter, J. Phys. A: Math. Theor. 41, 224019 (2008).

[2] D.-H. Kim and A. E. Motter, New J. Phys. 10, 053022 (2008).

[3] B. Danila, Y. Yu, J. A. Marsh, and K. E. Bassler, Phys. Rev. E 74, 046106 (2006).

[4] B. Danila, Y. Sun, and K. E. Bassler, Phys. Rev. E 80, 066116 (2009).

[5] G. Yan, T. Zhou, B. Hu, Z.-Q. Fu, and B.-H. Wang, Phys. Rev. E 73, 046108 (2006).

[6] M. Tang and T. Zhou, Phys. Rev. E 84, 026116 (2011).

[7] R. Guimerà, A. Arenas, A. Díaz-Guilera, F. Giralt, Phys. Rev. E 66, 026704 (2002).

[8] W.-X. Wang, B.-H. Wang, C.-Y. Yin, Y.-B. Xie, and T. Zhou, Phys. Rev. E 73, 026111 (2006).

[9] C.-Y. Yin, B.-H. Wang, W.-X. Wang, T. Zhou, and H.-J. Yang, Phys. Lett. A 351, 220 (2006).

[10] S. Sreenivasan, R. Cohen, E. Lopez, Z. Toroczkai, and H. E. Stanley, Phys. Rev. E 75, 036105 (2007).

[11] C.-L. Pu, S.-Y. Zhou, K. Wang, Y.-F. Zhang, and W.-J. Pei, Physica A 391, 866 (2012).

[12] W.-X. Wang, C.-Y. Yin, G. Yan, and B.-H. Wang, Phys. Rev. E 74, 016101 (2006).

[13] B. Kujawski, G. J. Rodgers, and B. Tadić, Lect. Notes Comput. Sci. 3993, 1024 (2006)

[14] P. Echenique, J. Gómez-Gardeńes, and Y. Moreno, Phys. Rev. E 70, 056105 (2004)

[15] P. Echenique, J. Gómez-Gardeńes, and Y. Moreno, Europhys. Lett. 71, 325 (2005).

[16] X. Ling, M.-B. Hu, R. Jiang, and Q.-S. Wu, Phys. Rev. E 81, 016113 (2010).

[17] V. Tintor and J. Radunović, Photonic Network Communications 18, 55 (2009).

[18] D. Wischik, In ECOC, Scotland (2005).

[19] D. Wischik and N. McKeown, ACM CCR 35(2), 75 (2005).

[20] G. Raina, D. Towsley, and D. Wischik, ACM CCR 35(2), 79 (2005).

[21] M. Enachescu et al., ACM CCR 35(2), 83 (2005).

[22] M. Enachescu et al., In IEEE INFOCOM, Spain (2006).

[23] S. Gorinsky, A. Kantawala, and J. Turner, In ISCC, Spain (2005).

[24] S. Gorinsky, A. Kantawala, and J. Turner, Simulation 83(3), 245 (2007). 
[25] E. W. M. Wong, L. L. H. Andrew, T. Cui, B. Moran, A. Zalesky, R. S. Tucker, M. Zukerman, J. Lightwave Technol. 27(14), 2817 (2009)

[26] A.-L. Barabàsi, R. Albert, Science 286, 509 (1999).

[27] S.N. Dorogovtsev, J.F.F. Mendes, Evolution of Networks, Oxford University Press, Oxford, 2003.

[28] A.F. Rozenfeld, R. Cohen, D. ben-Avraham, S. Havlin, Phys. Rev. Lett. 89, 218701 (2002).

[29] http://www.surfnet.nl

[30] http://www.renater.fr

[31] https://www.uninett.no

[32] http://www.rediris.es

[33] E. W. Dijkstra, Numer. Math. 1, 269 (1959).

[34] K.-I. Goh, B. Kahng, and D. Kim, Phys. Rev. Lett. 90, 058701 (2003).

[35] S. Havlin and R. Cohen, Complex Networks - Structure, Robustness and Function, Cambridge University Press, Cambridge, 2010.

[36] R. Cohen and S. Havlin, Phys. Rev. Lett. 87, 278701 (2001). 